\title{
Asymmetric Game Dynamics in Complex Networks against Cascading Failures
}

\author{
Chenxi Shao \\ Department of Computer Science and Technology, \\ University of Science and Technology of China, \\ Anhui Province Key Laboratory of Software in \\ Computing and Communication, \\ Hefei, P.R.China. \\ cxshao@ustc.edu.cn
}

\author{
Yang Wang \\ Department of Computer Science and Technology, \\ University of Science and Technology of China \\ Hefei, P.R.China. \\ wangy12@mail.ustc.edu.cn
}

\begin{abstract}
Information asymmetry is one of the most important dynamic properties of complex networks. It has important significance to explore the effect of information asymmetry on cascading failures for realizing cascading dynamic properties. In this paper, after analyzing the information asymmetry during the load redistribution, asymmetric game theory has been constructed to describe the behavior of game among nodes during the load redistribution. And on the basis of this framework, a new cascading model is proposed in which the network robustness is mostly influenced by initial load of nodes and profit asymmetry ratio. The simulation results show initial load has a positive correlation with the cascading propagation and exists an intermediate value guaranteeing the minimum of the tolerance threshold, while the results of profit asymmetry ratio are on the contrary. These research results effectively demonstrate the value of information asymmetry theory on network security and provides new perspective to investigate the cascading failure dynamics.
\end{abstract}

Keywords-information asymmetry; asymmetric game; prisoner's dilemma game; cascading failures; complex networks

\section{INTRODUCTION}

After finding the features of small-world [1] and scalefree [2] of real networks, a number of network models are constructed to investigate different statistical and dynamical properties in different real systems. The statistical and dynamical properties have significant influence on the field of the network safety. An important class of dynamical processes is the so-called cascading failure dynamics, addressing how the failure of a node or a small part ofnodes in a network induces other nodes failure over the network. Owing to existing a large amount of the load in networks and the limited capacity of the node, cascading failures are common phenomena in many infrastructure networks, such as, the largescale blackouts in power grids [3], the internet collapse [4] and the traffic congestion [5]. To minimize the effect of cascading failures, many cascading models have been proposed [6-9]. The above cascading models put more emphasis on the effect of topology and load distribution on cascading propagation and treat cascading failures as static behaviors. However in real networks, information transmission among nodes is a kind of dynamic selforganizing process. There exists competitive behaviors during the load redistribution among nodes to keep themselves safty.

In the process of load redistribution, each node wants to maintain its normal and efficient functions by maximizing its output load and minimizing extra input load from its neighbors. In this competitive process, if the transferred load of a node is treated as payoff, the load redistribution in the network is equivalent to an evolutionary game [10]. During the process of the information redistribution, to avoid the reallocated load excessively concentrating and causing cascading overload, all node pairs should share the extra load mutually so that the total profit can be maximized; but for each node in the pair, the best strategy to maintain the maximum of its functioning is to reject the extra reallocated load from neighbors. So it is evident that there exists the similar dilemma in the process of information asymmetry compared with PDG.

Considering the cascading propagation highly depends on the load redistribution, thus to investigate the effect of information asymmetry on network robustness becomes a signicant issue. To this end, we introduce the theory of asymmetric game to describe the competitive behaviors during the load redistribution. Then based on the above theory, a cascading model is proposed to simulate the competitive behaviors among nodes.

\section{MODEL}

\section{A. The definition of information asymmetry theory}

The information asymmetry theory uses the paradigm of prisoners dilemma game to study the self-organized behaviors in the load redistribution. Nodes connected in a pair are players in the game. Every player can choose either to cooperate(C) or to $\operatorname{defect}(\mathrm{D})$. When both nodes in a pair are cooperators, each of them is offered a reward $R$ for being able to transmit load to the partner during the redistribution; if one of them decides to defect for not receiving extra reallocated load, the defector can get higher payoff while the cooperator gets lower. Following previous models addressing cascading problems [6,11], initial load of a node $i$ whose degree is $k_{i}$ is assumed to be $L_{i}=k_{i}^{\alpha}$, where $\alpha$ is a tunable parameter to control the strength of initial load on the node $i$. Since the node capacity on real-life networks is generally limited by cost, it is natural to assume that the capacity $C_{i}$ of node $i$ is 
proportional to its initial load and defined as $C_{i}=\gamma * L_{i}$, where the constant $\gamma$ is the tolerance parameter characterizing the tolerance of the network. After a node fails, if its neighboring nodes have the enough capacities to handle the extra reallocated load, the network will remain a stable state and cascading failures will not occur. Therefore, the reward of cooperator $i$ with its neighbor $j$ is defined as:

$$
R_{i}=L_{i} \cdot \frac{C_{j}}{\sum_{m \in \Gamma_{i}} C_{m}},
$$

where $\Gamma_{i}$ is the set of neighboring nodes of $i$. The reward of $i$ quantifies the node's ability to transmit load to $j$ during the redistribution, which is not only determined by $i$ 's initial load but also determined by the capacity of the neighbor $j$. The higher the reward is, the more load the partner $j$ can handle in the process of load redistribution, which makes $i$ maintain its function when the congestion comes. However, the cooperator $i$ may preferentially change the strategy to defection for the reallocated load from cooperator $j$ is too much to be accommodated or it is too important to hold extra load. As the defection of $i$ implicates that cooperator $j$ receives reallocated load from $i$ while $i$ rejects $j$ 's, so the temptation $(\mathrm{T})$ of defection is higher than the reward $(\mathrm{R})$ of cooperation. Considering the mutual cooperation makes a node pair become more capable of handling reallocated load than the defection does in a real network, the temptation of a defector is quantified by $\beta \cdot R$. For simplicity, Nowak [12] rescaled the payoffs by setting $T=b, R=1$ and $P=S=0$, where $1 \leq b \leq 2$ ensures a proper payoff ranking and preserves the essential dilemma between individual profits and welfare of the population for repeated games. Without loss of generality, the payoff matrix of information asymmetry is defined as:

$$
\begin{array}{ccc} 
& C & D \\
C & R & 0 \\
D & \beta R & 0
\end{array}
$$

According to this payoff matrix, when node $i$ and node $j$ in a pair are both mutual cooperators, the reward of $i$ is $R_{i}$ while the reward of $j$ is $R_{j}$; however, when node $i$ defects while the other cooperates, the temptation of $i$ is $\beta R_{i}$ while the sucker's payoff of $j$ is 0 . The payoff of a node in the process of information redistribution represents its ability of handling the reallocated load. The higher the payoff is, the stronger the ability is, which leads the node less to overload. From above definition, it can be seen that when the topology of the network is fixed, the information asymmetry is determined by $\alpha$ and $\beta$. The parameter $\alpha$ controls the strength of initial load of nodes and determines the value of initial load; the parameter $\beta$ represents the profit asymmetry ratio and determines the difference of profits between cooperators and defectors during the information redistribution.

\section{B. The definition of a cascading model based on the asymmetric game}

Considering the effect of the load redistribution on the network robustness, a cascading model is proposed based on the information asymmetry theory. Our aim is to investigate how to adjust initial load and profit asymmetry ratio to maximize the network robustness against cascading failures. The set of players involved in the game is consist of all survived nodes in the network. At each time step, the actual payoff gained by any individual is the sum of payoffs resulting from all interactions with others.Initially, each node is designated either as a cooperator or a defector with equal probability. Then there are two dynamical processes. Thus, the information asymmetry can be used to describe the load dynamical allocation and its determinants include initial load and profit asymmetry ratio.

1). Asymmetric game playing and payoffs. The payoff of each node is initialized to be $\varepsilon$ which is positive but significant below unity. The reason will be explained soon afterwards. At each time step (iteration), each node $i$ plays the game with all its neighbors and gets the payoff by Eqs.(1). The accumulated payoff of node $i$ in each iteration is $P_{i}$. After all node-pair games finished in an iteration, each node $i$ randomly chooses a survived neighbor $j$ and adopts its strategy $s_{j}$ in accordance with the probability [13]:

$$
P\left(s_{i} \leftarrow s_{j}\right)=\frac{1}{1+e^{\left(P_{i}-P_{j}\right) / \kappa}},
$$

where $\kappa$ quantifies uncertainty of strategy adoption [14]. The value of $\kappa$ is simply fixed to be $\kappa=0.1$ in this paper so that the results have better recognition. After several iterations, the cooperation density andthe average payoff of the network will become steady(see Fig .1). The final accumulated payoff $\bar{P}_{i}$ of node $i$ is defined as its ability which can transmit the reallocated load to neighbors for avoiding congestion.

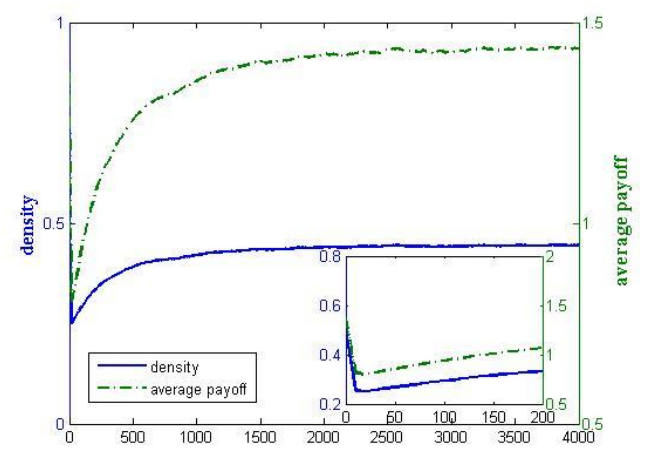

Figure 1. Time series of the cooperation density and average payoff on each node of the BA scale-free network with $N=5000,<k>=6$ for $\alpha=0.5$ and $\beta=1.4$. The inset is a part from time $=0$ to time $=200$. 
2). Failures and overloaded removal. The cascading propagation firstly originates from the redistribution of the load on failed nodes under attacks. At each iteration, the failed nodes will be added to the removing queue. In process 1 , after multiple repeated games, the ability of transmitting reallocated load of a node can be represented by its payoff. For the difference of the nodes' abilities, the preferential redistribution principle of the load is widely adopted to better describe the dynamic process of the load propagation. In this sense, when a node $i$ is removed, the load on it will be redistributed to its neighbor $j$ by the preferential probability. The additional load $L_{j i}$ received by node $j$ from node $i$ is:

$$
L_{j i}=L_{i}(t) \cdot \frac{\bar{P}_{j}}{\sum_{m \in \Gamma_{i}} \bar{P}_{m}} .
$$

as the initial payoff for each node has been set $\varepsilon$, so $\sum \bar{P}_{m}$ will never be 0 . if $L_{j}+L_{j i}>C_{j}$, it indicates that node $j$ has been overloaded and has to be added to the removing queue. The process of the cascading propagation above will be repeated until the removing queue is empty.

\section{THE RESULTS AND ANALYSIS OF THE CASCADING MODEL}

The topological structure of a network has a great influence on its function. Considering the scale-free networks are ubiquitous in nature and human society, we adopt the well-known BA scale-free network [2] as the physical infrastructure on top of which a cascading process takes place. BA networks can be constructed as follows: starting from $m_{0}$ fully connected nodes, a new node with $m\left(m \leq m_{0}\right)$ edges is added to the existing network at each
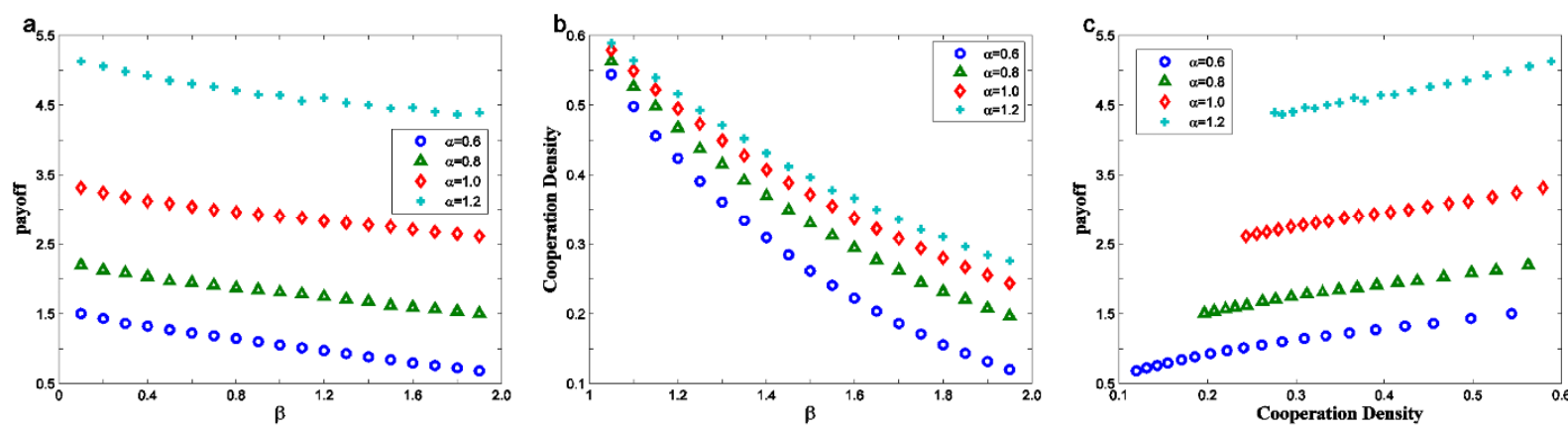

Figure 2. (a) the average payoff on each node and (b) the fraction of cooperators of the BA scale-free network as functions of parameter $\beta$ for different values of $\alpha$. (c) Demonstration of the correlation between the average payoff from (a) and the fraction of cooperators from (b).

Next, we explore the impact of initial load and profit asymmetry ratio on the cascading propagation. In order to measure the consequence created by the cascading propagation in a network, the average avalanche size $C F_{n}$ is adopted to quantify the network robustness against cascading failures and defined as: time step according to the preferential attachment, i.e., the probability of being connected to the existing node $i$ is proportional to degree $k_{i}$ of the existing node. In the numerical simulations, the network size is fixed as $N=5000$ and the parameters are set to be $m_{0}=3, m=3$, i.e., the average degree $\langle k\rangle$ is about 6 .

To begin with, we first examine how initial load $\alpha$ and profit asymmetry ratio $\beta$ influence the cooperation density and the average payoff of the network during the information redistribution. Fig .2(a) features the number of cooperators for different values of $\beta$ in four cases of $\alpha=0.6, \alpha=0.8, \alpha=1.0$ and $\alpha=1.2$. Every curve is obtained by averaging over 1000 different realizations on independent networks. It can be observed that no matter what the value of $\alpha$ is, the higher the value of $\beta$, the less the number of cooperators; however, when the value of $\beta$ is given, the higher the value of $\alpha$, the larger the number of cooperators. The correlation between $\alpha, \beta$ and the average payoff is presented in Fig .2(b). The results are similar to Fig .2(a)'s, i.e., the higher(lower) the value of $\alpha(\beta)$, the more the average payoff. Hence, during the process of information redistribution, it is clear that the high initial load promotes the emergence of cooperation and the increase of the average payoff, while the high profit asymmetry ratio impedes. Comparing the results of Fig .2(a) and Fig .2(b), it can be found the average payoff is directly proportional to the cooperation density(see Fig .2(c)), which well validates the dilemma in the information redistribution among nodes, i.e., although defection optimizes the individual payoff, mutual cooperation could yield a higher collective benefit. This result well validates the situation in information asymmetry is consistent with that in real networks. 
$0 \leq C F_{n} \leq N-1$. In Fig .3, by the capacity parameter $\gamma$, we compare the average avalanche size $C F_{n}$ of the failed nodes for different values of $\alpha$ and $\beta$. Every curve is obtained by averaging over 1000 different realizations on independent networks. It can be seen that there is a relatively great difference between $\alpha$ and $\beta$ to the network robustness against cascading failures. In Fig .3(a), for a given value of $\beta$, the higher the value of $\alpha$, the faster the $C F_{n}$ declines. In Fig .3(b), for a given value of $\alpha$, the lower the value of $\beta$, the faster the $C F_{n}$ declines. These findings indicate when the cascading occurs, the higher initial load is, the less the failed nodes are created; however, the higher profit asymmetry ratio is, the more the failed nodes are created.

From above simulations, it can be realized $\gamma$ controls the whole capacities of all nodes in the network. Apparently, if the value of $\gamma$ is large enough, then all nodes have larger extra capacities to handle the load under whatever attack, no cascading failure occurs. However, it is not realistic for the cost. Once the value of $\gamma$ is small enough, the cascading failure emerges because the capacity of each node is limited, sweeping the whole or part network to stop working. Hence, it makessense to select the smallest value of the $\gamma_{c}$ to protect the network at the lowest cost. For any curve in Fig .3, the responses of BA networks display threshold-like behaviors, namely, the critical threshold $\gamma_{c}$. The value $C F_{n}$ falls steeply when the value $\gamma$ increases to $\gamma_{c}$, and then remains a stable and low value, i.e., no cascading failure emerges and BA networks maintain their normal and efficient functioning. Therefore, a natural question arises whatthe relationship is between the critical threshold $\gamma_{c}$ and initial load $\alpha$ and redistribution $\beta$ in Fig .3. To better observe the effect of $\alpha, \beta$ on network robustness against cascading failures, by numerical simulations, we compare $\gamma_{c}$ under given values of $\alpha(\beta)$ for different values of $\beta(\alpha)$ in Fig .4. As can be seen in Fig .4(a), no matter what the values of the parameter $\beta$ are,all the values of $\gamma_{c}$ will be minimum when $\alpha=1$. For the range of $\alpha<1, \gamma_{c}$ in each curve decreases with the increase of $\alpha$, but its relationship with $\beta$ is not clear. While for the range of $\alpha>1, \gamma_{c}$ has a positive correlation with $\alpha$, that is the same with the values of $\beta$. As shown in Fig .4(b), no matter what the values of the parameter $\alpha$ are, $\gamma_{c}$ monotonically increases with $\gamma$ and it increasesmore steeply when $\alpha$ decreases. Therefore, it can be derived that there exists the optimal value of initial load at which the cascading is hardest to occur, while the value of the cascading critical threshold increases monotonically with the value of profit asymmetry ratio. In addition, comparing Fig .4(a) and Fig .4(b), it can be found that when the value of $\alpha$ is far from $\alpha=1$, the value of the critical threshold $\gamma_{c}$ is sensitive with profit asymmetry ratio $\beta$; while the value of $\alpha$ is close to $1, \beta$ rarely influences the value of the critical threshold $\gamma_{c}$.

Above all, in the process of information asymmetry, the high initial load impedes the cascading propagation, while the result of profit asymmetry ratio is opposite; furthermore, there exists an intermediate value of initial load that could guarantee the minimum critical threshold of cascading, while the value of the critical threshold increases linearly with profit asymmetry ratio.
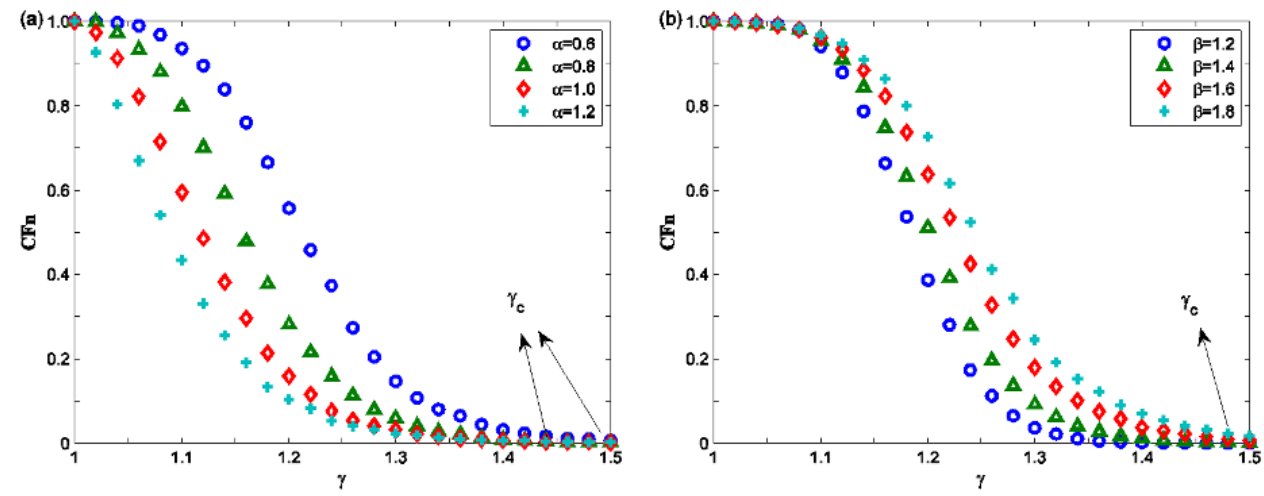

Figure 3. the average avalanche size $C F_{n}$ as functions of parameter $\gamma$ for (a) different values of $\alpha$ and (b) different values of $\beta$. 

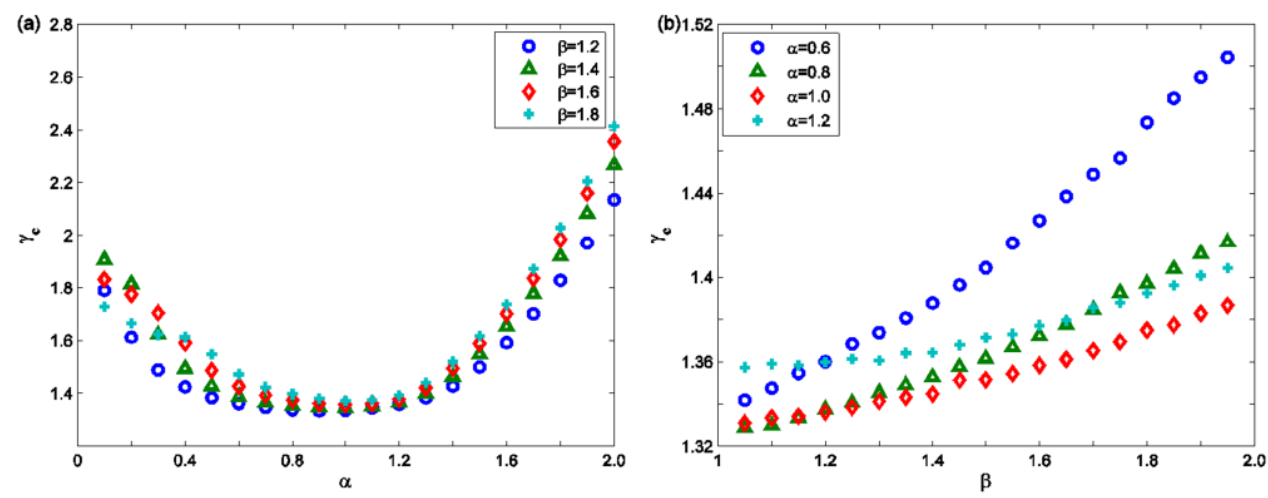

Figure 4. the critical threshold $\gamma_{c}$ as functions of (a) parameter $\alpha$ for different values of $\beta$ and (b) parameter for different values of $\alpha$.

\section{CONCLUSION}

In conclusion, we analyze the phenomenon of information asymmetry during load redistribution and construct a cascading model of asymmetric game to describe self-organizing competitive behaviors among nodes. It can be found that the results of the cascading model are determined by some tunable parameters: initial load and profit asymmetry ratio. The simulations show the high initial load impedes the cascading scale while the result of profit asymmetry ratio is opposite; furthermore, there exists an intermediate value of initial load guaranteeing the minimum of the tolerance threshold, while the tolerance threshold increases linearly with profit asymmetry ratio. These findings effectively demonstrate the value of information asymmetry theory on network security and give the important theory for emergent response and control of cascading failures under the major disaster.

\section{ACKNOWLEDGMENT}

This work is supported by the National Natural Science Foundation of China (Grant No.61472381 and 61174144).

\section{REFERENCES}

[1] Duncan J Watts and Steven H Strogatz. Collective dynamics of small-worldnetworks. nature, 393(6684):440-442, 1998

[2] Albert-Laszlo Barabasi and Reka Albert. Emergence of scaling in random networks. science, 286(5439):509-512, 1999.

[3] Yakup Koc, Martijn Warnier, Piet Van Mieghem, Robert E Kooij, and Frances MT Brazier. The impact of the topology on cascading failures in a power grid model. Physica A: Statistical Mechanics and its Applications, 402:169-179, 2014.

[4] JianweiWang, Chuan Zhang, Yi Huang, and Chong Xin. Attack robustness of cascading model with node weight. Nonlinear Dynamics, pages 1-12, 2014.

[5] Zhiyun Zou, Yao Xiao, and Jianzhi Gao. Robustness analysis of urban transit network based on complex networks theory. Kybernetes, 42(3):383-399, 2013.

[6] Adilson E Motter and Ying-Cheng Lai. Cascade-based attacks on complex networks. Physical Review E, 66(6):065102, 2002.

[7] Jan Oystein Haavig Bakke, Alex Hansen, and Janos Kertesz. Failures and avalanches in complex networks. EPL (Europhysics Letters), 76(4):717,2006.

[8] Dominik Heide, Mirko Sch afer, and Martin Greiner. Robustness of networks against uctuation-induced cascading failures. Physical Review E, 77(5):056103, 2008.

[9] Jorg Lehmann and Jakob Bernasconi. Stochastic load-redistribution model for cascading failure propagation. Physical Review E, 81(3):031129, 2010

[10] John Maynard Smith. Evolution and the Theory of Games. Cambridge university press, 1982.

[11] Jianwei Wang, Lili Rong, Liang Zhang, and Zhongzhi Zhang. Attack vulnerability of scale-free networks due to cascading failures. Physica A: Statistical Mechanics and its Applications, 387(26):6671-6678, 2008.

[12] Martin A Nowak and Robert M May. Evolutionary games and spatial chaos. Nature, 359(6398):826-829, 1992.

[13] Han-Xin Yang, Zhi-Xi Wu, and Bing-Hong Wang. Role of aspiration induced migration in cooperation. Physical Review E, 81(6):065101, 2010

[14] Jesus Gomez-Gardenes, M Campillo, LM Flora, and Yami Moreno. Dynamical organization of cooperation in complex topologies. Physical Review Letters, 98(10):108103, 2007. 